\title{
De la théorie à la pratique : appliquer des modèles cognitifs de la motivation dans un centre de langues
}

Denyze Toffoli

\section{OpenEdition}

Journals

Édition électronique

URL : http://journals.openedition.org/asp/1198

DOI : $10.4000 /$ asp. 1198

ISBN : 978-2-8218-0394-7

ISSN : 2108-6354

\section{Éditeur}

Groupe d'étude et de recherche en anglais de spécialité

Édition imprimée

Date de publication : 1 novembre 2003

Pagination : $99-114$

ISSN : 1246-8185

Référence électronique

Denyze Toffoli, « De la théorie à la pratique : appliquer des modèles cognitifs de la motivation dans un centre de langues », ASp [En ligne], 41-42 | 2003, mis en ligne le 28 avril 2010, consulté le 19 avril 2019. URL : http://journals.openedition.org/asp/1198; DOI : 10.4000/asp.1198

Ce document a été généré automatiquement le 19 avril 2019

Tous droits réservés 


\title{
De la théorie à la pratique : appliquer des modèles cognitifs de la motivation dans un centre de langues
}

\author{
Denyze Toffoli
}

\section{Définition}

1 Tout comme l'aptitude, la motivation est un concept qui a suscité beaucoup d'attention en apprentissage des langues et dans le domaine de la pédagogie de façon générale. Dörnyei (1994 : 237) affirme : «motivation is one of the main determinants of second / foreign language $\left(L_{2}\right)$ learning achievement». Même dans le langage courant, la notion de motivation ressort régulièrement lorsqu'on parle d'apprentissage, souvent liée à un rejet de responsabilité si l'apprentissage ne se passe pas bien. C'est le cas des apprenants qui accusent les enseignants de ne pas être très motivants ou des formateurs qui insistent sur le fait que les stagiaires « ne sont pas du tout motivés ».

2 Skehan (1989), rappelle quatre des plus importantes dimensions de la motivation : qu'elle soit interne ou externe à l'individu, inhérente ou résultant du contexte de l'apprentissage. Nous retrouverons ces dimensions dans la plupart des théories scientifiques de la motivation, même si O'Bryen précise que : « researchers do not yet agree about whether or not a set of items can be established that define the motivation construct " (1996: 75).

3 La motivation est un facteur déterminant dans tous les domaines de la vie, que ce soit le travail, les relations interpersonnelles ou le loisir. Vallerand et Thill la définissent ainsi :

[...] le concept de motivation représente le construit hypothétique utilisé afin de décrire les forces internes et/ou externes produisant le déclenchement, la direction, l'intensité et la persistance du comportement. (1993:18)

4 Le déclenchement concerne l'énergie mise en œuvre pour démarrer ou dynamiser l'action. La direction, c'est la canalisation de cette énergie pour diriger le comportement 
en question vers le but recherché. L'intensité représente la manifestation de la motivation dans le comportement : plus un sportif désire gagner une compétition, plus il s'entraînera (physiquement et mentalement). La persistance du comportement indique la durée et l'évolution de la motivation dans le temps, pour aller jusqu'au bout de l'action entreprise.

\section{Le centre de langues en entreprise}

Les personnes et exemples décrits ci-dessous ont été rencontrés et observés dans un centre de langues au sein d'une entreprise française sur une période de seize mois. Il s'agissait d'un centre de langues créé dans le cadre d'un contrat commercial, soumis à la législation sur la formation continue. L'entreprise commanditaire est la filiale d'une grande entreprise américaine dans le secteur des services. Le siège européen est situé en Angleterre et la spécialisation des sites a provoqué des contacts de plus en plus fréquents entre salariés situés dans différents pays européens, ainsi qu'avec la maison mère.

Le Centre de langues se voulait un centre d'autoformation éducative (Carré 1997), où l'apprentissage «centré sur l'apprenant» (Nunan 1995) était rendu explicite par l'organisation même des apprentissages. Une réflexion sur l'autonomie, l'autodétermination et l'individualisation avait amené à la mise en place d'un système de formation individualisée, sur la base d'un entretien d'accès à la formation (positionnement de niveau, détermination des objectifs, découverte des préférences cognitives, élaboration du parcours personnel, mise à jour des motivations, etc. - cf. Toffoli 2000: 249-267 et 362-381). La détection des motivations individuelles des apprenants devait permettre de calquer toute la formation sur ces éléments, que ce soit en termes de forme ou de contenu.

7 Pendant la période d'observation, 263 entretiens individuels (d'une durée moyenne d'une heure) ont débouché sur des parcours de formation. Cette première étape du cursus de formation (l'entretien) a permis d'apprécier, en termes quantitatif et qualitatif, les besoins de l'entreprise, ainsi que les besoins individuels, professionnels et personnels des apprenants. Pour chaque personne, le parcours de formation défini a pris en compte ses antécédents, ses préférences cognitives et affectives, ses motivations, ses besoins professionnels et extra-professionnels, son emploi du temps et ses variations d'activité.

Une sélection de trente apprenants a suivi un parcours " complet », c'est-à-dire est passée par toutes les étapes du diagnostic initial à une validation finale. L'objet de cet article est d'analyser le concept de la motivation et son utilisation dans un tel cadre. Ainsi, c'est à partir des histoires de ces trente personnes que les propos théoriques seront illustrés. $\mathrm{Ce}$ sont des histoires d'adultes très différents, avec des acquis, des expériences, des objectifs et des besoins d'apprentissage divers, ainsi que des modes de fonctionnement personnel et des paramètres cognitifs, affectifs et métacognitifs uniques pour chacun d'entre eux.

9 Les apprenants représentent une panoplie diverse de pratique et d'expérience de l'anglais. Quelques apprenants n'avaient que très peu d'expérience de l'anglais ; d'autres avaient fait des études universitaires en anglais et utilisaient la langue presque quotidiennement. Quelques-uns étaient très timides et presque tétanisés par l'utilisation orale de l'anglais; certain de leurs collègues plus extravertis étaient prêts à dialoguer avec tout le monde à tout moment. Leurs âges variaient de 25 à 60 ans. Un peu plus de la 
moitié (seize sur trente) étaient des femmes. Certains dans le groupe n'avaient jamais essayé d'apprendre une autre langue, d'autres en parlaient trois ou quatre.

C'est en partie cette grande diversité qui distingue cette population d'apprenants d'une population d'élèves, ou encore d'étudiants (où les âges sont très rapprochés, les antécédents scolaires similaires, les objectifs scolaires ou professionnels ressemblants...). Là où des étudiants ont tous réussi certaines épreuves scolaires (ils ont tous eu le baccalauréat), les niveaux d'études dans cet échantillon allaient du plus faible (arrêt de scolarité avant le CAP) à des études supérieures importantes (ingénieurs et équivalents).

Ils sont néanmoins tous salariés d'une même entreprise avec sa propre culture et sa propre personnalité, minimisant (au moins en apparence) la diversité socioculturelle des individus. L'échantillon de 30 apprenants étudié n'est pas aléatoire, même s'il est à la limite de la représentativité sur un plan statistique. Que représentent ces trente individus? Les deux cent soixante personnes qui étaient inscrites en formation langues? Les milliers de salariés d'autres entreprises engagés dans des cursus de formation similaires? S'il est valable de supposer une certaine représentativité dans la première hypothèse (les inscrits), chaque écart par rapport au contexte spécifique de l'étude falsifie un peu plus toute représentativité qu'on peut déclarer. Mais il ne s'agit pas ici d'une expérimentation quantitative, même si quelques réflexions de type quantitatif apporteront des informations complémentaires utiles. L'organisation même du texte présente des applications et pistes de réflexion par rapport à chaque théorie, plutôt que d'établir un cadre théorique, décrire les actions expérimentales et analyser les résultats. C'est au moyen des histoires individuelles et d'exemples contextualisés, avec des applications pédagogiques possibles, que cet article tente d'illustrer la mise en application de théories de la motivation dans un centre de langues.

\section{Quatre théories pour le centre de langues}

Deux grandes orientations divisent la quarantaine de théories (Vallerand \& Thill 1993) composant la psychologie de la motivation: l'une considère la personne comme un être passif qui subit la motivation et l'autre estime qu'elle interagit activement avec l'environnement (Vallerand \& Blanchard 1998). Ce sont des théories et recherches issues de ce deuxième courant qui seront examinés ici, car dans un centre de langues, où l'objectif est « learner-centered learning » (Nunan 1988, 1995), c'est l'action de l'individu qui aura l'influence la plus déterminante sur son apprentissage. L'implication de ce choix n'est pas forcément la plus facile, car on ne peut plus mettre toute la responsabilité de la motivation sur le compte des formateurs ou institutions, comme on aurait pu le faire dans une perspective purement comportementaliste (tel stimulus produirait telle motivation). Une telle théorisation met la responsabilité de la motivation quelque part entre le « tout externe » du comportementalisme, le «tout inconscient » de la psychanalyse et le « tout déterminé personnellement » de l'humanisme, là où chacun des acteurs de la formation, chacun des sous-systèmes de cette structure est partiellement responsable de la motivation, mais où la responsabilité de l'individu, de l'apprenant est primordiale. Ce choix épistémologique implique également une multi-dimensionnalité qui rend l'analyse de la motivation plus complexe, mais par cela-même, de toute évidence, plus proche de la réalité (Williams 1994). Comme l'aptitude ou le style d'apprentissage, la motivation est une notion composite qui, selon les auteurs, englobe plusieurs paramètres cognitifs, 
affectifs ou sociaux. Ainsi, dans la mise en œuvre pratique, il faut toujours garder à l'esprit l'aspect global de l'apprentissage.

Dans le cadre de la formation dans un centre de langue, quatre théories de la motivation semblent être particulièrement utiles, à la fois pour expliquer les comportements des apprenants et pour indiquer des modalités d'organisation ou d'animation/enseignement (Vallerand 1993 ; Carré 1997). Ce sont: les théories de l'autodétermination et, par extension, de l'évaluation cognitive de Deci et Ryan $(1985,1991)$; la théorie des buts (Nicholls 1984 ; Ames 1992) ; la théorie de l'attribution (Heider 1958; Weiner 1979, cités dan Vallerand \& Thill 1993) et celle de l'auto-efficacité de Bandura (1986). Les travaux de Gardner (1981, 1992, 1993, 1994, 1995) et son modèle socio-éducatif, bien qu'ils représentent l'un des plus grands corps de recherche sur la motivation dans le domaine de la RAL, ne seront pas examinés ici, car, comme constate Dörnyei: "Gardner's motivational construct does not include details on cognitive aspects of motivation to learn " (1994 : 273). Les quatre modèles choisis permettent d'accéder au fonctionnement cognitif et, à partir de là, de construire des plans d'action avec et pour les apprenants.

\section{Buts et objectifs}

Dans une perspective de type cognitif, la motivation est liée de près à la détermination des objectifs car c'est cette dernière qui influencera le comportement et ainsi exploitera les autres paramètres de la motivation. La théorie des buts indique que pour être moteur et mener aux plus grandes performances, un but doit être difficile à atteindre, spécifique et voulu (Thill 1993 : 396-399 ; Oxford \& Shearin 1994).

Les travaux d'Edwin Locke, résumant une étude longitudinale sur plus de 10 ans aux États-Unis (Locke et al. 1981), débouchent sur huit conclusions essentielles. Les trois premières confirment les liens entre la détermination des objectifs, la motivation (déclenchement, direction, intensité, persistance dans l'action) et la performance. Non seulement elles soulignent l'importance de la définition des objectifs, mais elles indiquent des critères pour décrire des objectifs de formation (spécifiques et vérifiables). Les quatre autres concernent la capacité, le feed-back, les récompenses (à utiliser à bon escient) et l'adhésion de l'individu aux objectifs (même s'il n'en est pas à l'origine).

La théorie du contrôle postule une hiérarchie de buts plus ou moins motivants (Vallerand \& Thill 1993). De ce point de vue, un objectif d'ordre comportemental concret (écouter la chaîne CNN le matin) est très bas dans la hiérarchie motivationnelle alors que celui d'ordre abstrait (être un bon professionnel, être fier de soi) est très élevé et donc beaucoup plus motivant. 


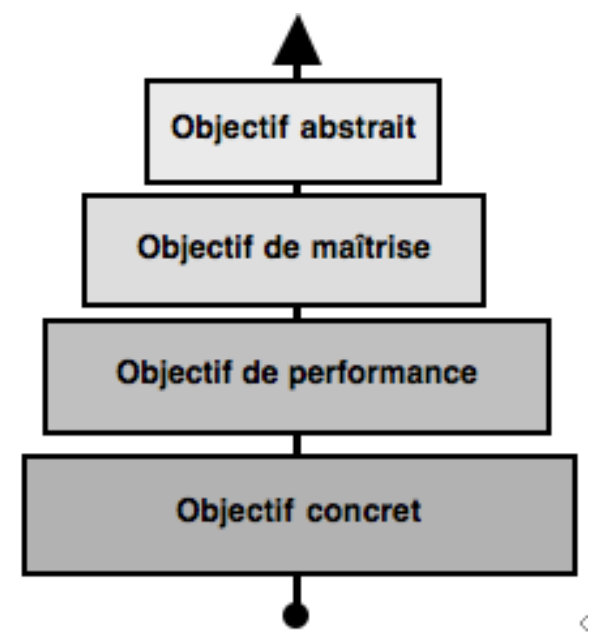

Oxford \& Shearin (1994 : 19) considèrent que :

goal setting can have exceptional importance in stimulating $\mathrm{L}_{2}$ learning motivation, and it is therefore shocking that so little time and energy are spent in the $\mathrm{L}_{2}$ classroom on goal setting.

C'est à la lumière de telles affirmations qu'un processus systématique de détermination d'objectifs a été mis en place dans le centre de langues. Un entretien d'orientation en formation langues a été crée, structuré autour de questions visant la détermination d'objectifs. Cette démarche, en sept étapes (sept questions) sera brièvement expliquée cidessous («l'entretien d'orientation»), afin de donner des exemples de l'application des théories des buts, en articulation avec les autres théories décrites.

Le but choisi, le comportement mis en œuvre pour le réaliser et l'impact effectif sur l'environnement vont amener l'individu à évaluer l'action qu'il a entreprise et, si elle correspond aux attentes, ramèneront soit à la détermination de nouveaux buts, soit à l'abandon de l'activité. L'atteinte d'un objectif (résultat conforme aux attentes) est souvent une raison suffisante d'arrêter une activité (cf. «J'ai réalisé mon voyage en Écosse, j'arrête de mettre de l'argent de côté»). Elle peut, par contre, provoquer la détermination de nouveaux buts, liés au premier (cf. « Mon voyage m'a tellement plu, je veux y retourner l'année prochaine pour deux mois»). Le renforcement positif des comportementalistes est ainsi toujours considéré comme un facteur de motivation important (Vallerand \& Thill 1993).

\section{V.I.E. et auto-efficacité}

19 Les théories de V.I.E. («valence » [valeur], instrumentalité, « expectation » [attente de réussite]) remontent aux recherches de E. Tolman et K. Lewin dans les années 1930 (Vallerand \& Thill 1993). Elles postulent que la motivation se construit sur deux fondements : d'une part la valeur que l'on accorde à l'objectif poursuivi, d'autre part l'attente de réussite concernant ce même objectif, c'est-à-dire la probabilité de pouvoir obtenir le résultat visé. De nombreuses études sur la motivation sont parties de ce cadre global de l'« expectation-valeur». V. Vroom (1964, cité dans Carré 1997) a précisé et élargi cette théorie, en y intégrant la notion d'instrumentalité, qui décrit la perception (subjective) de l'utilité de l'action à entreprendre pour réaliser l'objectif. 
20 Alfred Bandura a développé la notion de l'expectation en y introduisant le concept d'auto-efficacité, c'est-à-dire une confiance en soi, en ses capacités personnelles à mener le projet à bien et la probabilité de réussite. D'après Bandura (cité dans Carré 1997 : 72), «à mesure que s'élève l'auto-efficacité, les buts que les gens se fixent à eux-mêmes s'élèvent et leur engagement à apprendre s'affirme». Dans le domaine de l'activité professionnelle, le modèle V.I.E. de la motivation est, d'après R. Francès (cité dans Carré 1997 : 66), «le cadre conceptuel le plus solide, utilisé depuis trente ans presque sans changement, ce qui est un bon indice de son pouvoir prédictif ».

On peut cependant lui reprocher, comme le font Vallerand \& Thill (1993), de se fonder sur l'hypothèse qu'un sujet calculera vraiment la valence et l'expectation et prendra toujours une décision rationnelle en fonction de ses calculs. Dans le cadre d'une formation autodirigée en centre de langues l'apprenant aurait à jongler avec la distance entre les compétences langagières actuelles et désirées, le temps disponible, les contraintes extérieures et de nombreuses dispositions personnelles. Edgar Thill doute du fait que :

[...] l'individu puisse se livrer à des calculs relativement complexes en combinant de multiples informations ..., notamment dans le cas où des décisions rapides doivent être prises. (1993: 397)

C'est ainsi que l'entrée en jeu d'un participant externe - formateur ou consultant - peut s'avérer utile. Il assiste l'apprenant pour qu'une décision informée puisse être prise et assimilée.

Dans la formation en général, et par extension en RAL, le modèle V.I.E. permet de prédire notamment l'intensité et la persistance dans l'apprentissage. Dans le cadre d'un conseil en orientation, il permet de décrire un fonctionnement rationnel de la motivation auquel un apprenant peut choisir d'adhérer (ou non).

\section{Un entretien d'orientation}

24 Ainsi, en partant des théories des buts, de la V.I.E. et de l'auto-efficacité, un entretien individuel d'orientation en formation langues a été mis en place, permettant d'identifier, et peut être d'influencer, le déclenchement, la direction, l'intensité et la persistance dans l'apprentissage de l'anglais. Afin d'en comprendre son fonctionnement par rapport aux éléments théoriques, il sera décrit succinctement ci-dessous.

Ce questionnement n'est qu'un élément de l'entretien. Il vise à faire expliciter les objectifs professionnels (mais aussi personnels, car l'interaction des deux fait croître la motivation), à les détailler et à les décortiquer pour les rendre plus opérationnels et/ou plus accessibles. La réussite du déclenchement du processus d'apprentissage dans le centre de langues dépend en grande partie de la précision de cette étape.

Dans le contexte d'entreprise décrit ici, de nombreuses personnes sont déjà familiarisées aux démarches de détermination d'objectifs. Globalement, elles s'y impliquent facilement et rapidement, ayant peu besoin de répétition ou de reformulation des questions. Elles répondent souvent avec une formulation standardisée et facile à repérer pour l'interviewer.

Le questionnement comporte sept questions ou étapes. La formulation n'est pas toujours identique d'un entretien à l'autre, ce qui importe c'est que les réponses obtenues correspondent aux critères indiqués. Dans leur forme la plus brute, et la plus simple, les 
questions sont les suivantes (les commentaires entre parenthèses indiquent le type de renseignement recherché) :

- Que voulez-vous être capable de faire en anglais? (Objectifs concrets, liés à la situation professionnelle)

- Qu'est-ce que cela vous apportera? (Finalité, professionnelle ou personnelle)

- Comment saurez-vous que vous avez atteint vos objectifs? (Critères)

- Où, quand et avec qui voulez-vous l'obtenir? (Contexte)

- De quelles ressources (internes) disposez-vous qui peuvent vous aider? (Capacité)

- Qu'est-ce qui, jusqu'ici, vous a empêché d'atteindre vos objectifs en anglais ? (Contraintes)

- Que faut-il faire maintenant? (Déclencheur / Négociation de parcours)

Sans détailler les justifications théoriques pour chacune de ces questions, un commentaire serait de mise concernant l'adéquation entre les objectifs visés par l'entreprise et ceux de l'individu. Si Locke et ses collègues (1981) ont trouvé que des objectifs imposés (s'ils sont acceptés par l'individu) ont le même effet que les objectifs déterminés par l'individu lui-même, il est parfois difficile de faire accepter (devenir sien) des objectifs institutionnels de type «tous les techniciens doivent être capables de lire une notice et de suivre une formation technique en anglais ». Il est intéressant de noter que l'entreprise concernée par cette expérience, qui se veut à l'avant-garde de la gestion des ressources humaines et se positionne en tant qu'organisation apprenante, peut néanmoins fixer ce genre d'objectif, assorti de la sanction "sinon, il ne peut pas participer aux formations sur les nouveaux produits (qui ont lieu en anglais) » et (sousentendu) «par conséquent sa carrière s'arrête ».

Par contre, le constat de Locke est particulièrement important (et moins problématique) lorsque l'on est face à des personnes qui commencent une formation en langue pour la première fois, qui ne savent pas à quoi s'attendre, ni ce qui est possible en terme de progression. Dans ces cas, l'entreprise, via le formateur / conseiller, peut suggérer des objectifs qui risquent d'obtenir l'adhésion de l'individu.

A ce stade de l'entretien l'apprenant a pris conscience de la plupart des facteurs sousjacents à la motivation (à moins de résistances particulières). Il est alors prêt à prendre des décisions et à s'engager (ou non) sur un parcours de formation individuelle.

L'étape de la négociation du parcours reste à faire. Elle vise l'établissement d'un accord entre l'apprenant, l'équipe de formateurs et l'entreprise (même si cette dernière ne prend pas part à la négociation, elle ratifie les conclusions qui en résultent) sur le parcours de formation à suivre. Comme l'ensemble du processus, la négociation se veut dialogue dans le respect de toutes les parties, c'est-à-dire l'entreprise, l'apprenant et l'équipe de formateurs et cible un aboutissement " gagnant-gagnant-gagnant».

C'est une phase qui est souvent très courte, car, comme indiqué ci-dessus, l'apprenant trouve souvent, au moyen des questions qu'il est amené à se poser pendant l'entretien, des idées relativement précises sur la manière dont il veut (et peut) apprendre. Le rôle de l'interviewer à cette étape est de fournir des informations sur les possibilités qui sont disponibles dans le centre, suggérer des modalités de formation en dehors du centre qui soient en accord avec les contraintes, goûts et besoins de l'apprenant, préconiser les ateliers ou outils qui seront les plus aptes à amener l'apprenant à ses objectifs, en accord avec ses préférences cognitives et affectives. C'est une phase importante, car elle fixe souvent les énergies à mettre en œuvre chez l'apprenant. Ainsi elle agit pour déclencher la motivation. 


\section{L'attribution}

Si la motivation concerne en général une orientation vers l'avenir, la théorie de l'attribution porte un regard vers le passé et l'analyse des performances antérieures. Elle indique que, même en cas d'échec, la façon dont un individu analyse ses résultats et ce à quoi il les attribue va influencer sa motivation pour la suite. Le croisement de quatre perceptions possibles de réussite ou d'échec (stable ou instable ; interne ou externe) a abouti à l'identification de quatre causes de performance :

- la capacité, compétence ou aptitude (stable et interne)

- la difficulté de la tâche (stable et externe)

- l'effort (instable et interne)

- la chance (instable et externe) (Deschamps 1993).

Schéma 2. L'attribution

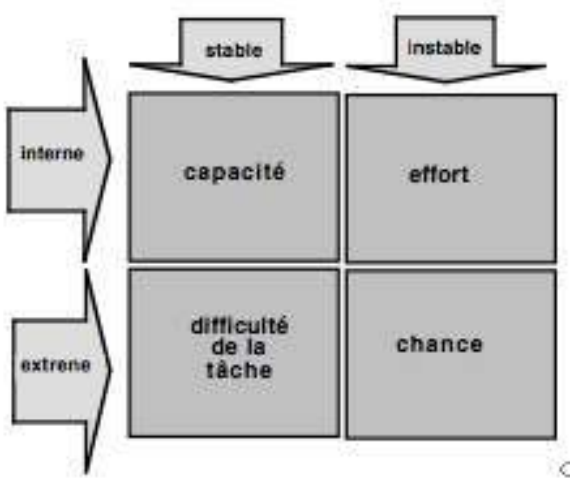

34 En fonction de la façon dont une personne perçoit ses résultats, et de ce à quoi elle les attribue, elle sera plus ou moins motivée pour continuer son apprentissage ou pour essayer à nouveau une tâche. L'attribution de la réussite à des facteurs internes contribue à la perception de compétence et à la persistance dans l'apprentissage. Ces perceptions vont en retour influencer positivement l'apprentissage, en augmentant sa potentiel de réussite. Une spirale de succès est ainsi enclenchée. «Anna " ${ }^{1}$, par exemple, attribue ses résultats à sa compétence personnelle (facteur interne et stable). Elle sait qu'elle peut s'appuyer sur cette compétence pour continuer à progresser. Cela est motivant en soi et l'équipe de formation n'a à veiller qu'à maintenir cette attitude (renforcer le message).

Nelson (1991), dans le cadre de l'apprentissage de la rédaction en anglais, observe « la spirale de la réussite » qui se met en place lorsque les apprenants ont les moyens de se rendre compte de leurs réussites, de les attribuer à des paramètres identifiables, et ainsi de modifier leurs attitudes (croyances) vis-à-vis du processus de l'écriture. 


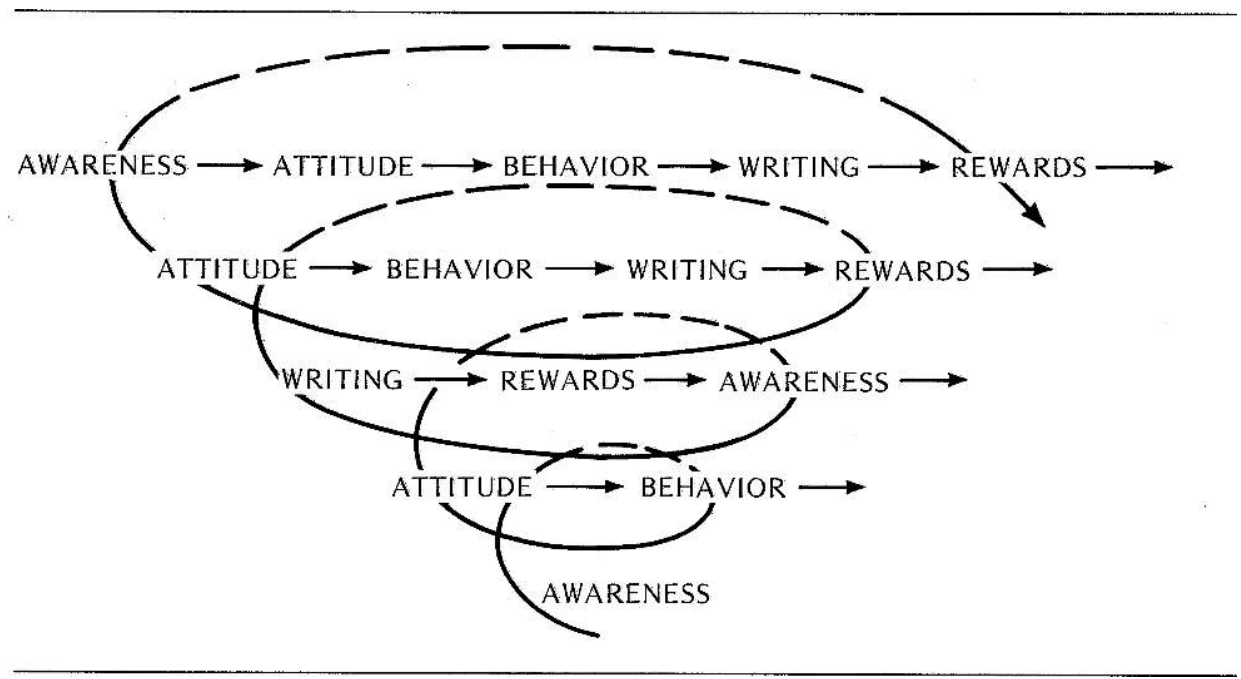
«Christine» attribue ses mauvais résultats en anglais à son manque de confiance. Elle considère cet état affectif comme immuable et permanent, et malgré une grande connaissance de l'anglais, elle a du mal à mettre cette connaissance en œuvre. Elle voit plus volontiers les résultats « limitants » (ne pas oser parler) que les résultats positifs (sa licence d'anglais). L'équipe de formation a essayé de faire prendre conscience à «Christine» d'abord de la qualité de ses connaissances, pour développer sa confiance, mais l'aspect « stable » de ce facteur d'attribution (exogène à l'apprentissage de l'anglais) en a limité la portée. néfaste, enclenchant une spirale négative de démotivation. Prenons le cas de "Jeanne ", qui attribue ses résultats en formation à son patron, qui peut à tout moment lui donner un travail de dernier moment qui l'empêche d'aller en cours d'anglais. "Jeanne" considère n'avoir aucun moyen d'action propre face à cette situation: elle subit. L'imprévisibilité de son patron lui permet de ne pas s'investir dans son apprentissage, d'éviter une prise en charge personnelle. Les formateurs se sont trouvés relativement désemparés face à une telle attitude. Leurs suggestions de réorganisation ont rencontré peu de succès et même le changement du patron de «Jeanne » en cours d'année n'a pas modifié la situation. Sans aller jusqu'à lui attribuer une mauvaise foi quelconque, on peut supposer que "Jeanne" trouvait son compte dans cette situation. Il reste à savoir pourquoi, malgré tout, elle voulait maintenir son inscription, plutôt que de se retirer du centre de langues.

«Valérie » attribue sa pratique récente de l'anglais à un facteur externe et instable aussi, mais pour elle ceci est un état temporaire, tenant au manque d'occasions de parler. Sa réponse est de s'inscrire en formation, se procurant ainsi les occasions désirées.

Les facteurs externes et stables peuvent être limitants ou facilitants, en fonction de la perception de l'apprenant: "Patrick» attribue ses mauvais résultats en anglais à la complexité de la langue elle-même. Cette attribution rend l'anglais particulièrement difficile pour lui . Afin de faciliter la tâche, les formateurs ont essayé de trouver avec lui des aspects de la langue qui étaient faciles (ou moins complexes qu'en français, par exemple). «Cathie » aussi attribue ses (bons) résultats en anglais à un facteur externe et 
stable, mais pour elle cela tient au fait que les cours «étaient faciles ». Insister sur la facilité de cet apprentissage lui permet de continuer à apprendre, sans effort insurmontable.

Enfin, même après un échec, les espoirs de réussite ultérieure sont motivants et peuvent influencer positivement l'apprentissage, si l'individu attribue son échec à une cause « interne et instable » - par exemple un manque d'implication personnelle (Vallerand \& Thill 1993). Cinq stagiaires de notre échantillon attribuent leurs résultats en anglais à l'effort et (parfois) à la disponibilité. Cette attribution leur permet de se re-motiver et d'augmenter leurs efforts lorsqu'ils veulent progresser. Le rôle des formateurs devient ainsi essentiellement un rôle de questionnement, afin d'explorer les différents moyens d'augmenter l'effort. Faut-il dégager du temps, avoir accès à des outils plus attrayants (attrayants de quelle façon), trouver d'autres organisations du travail...?

41 Les expériences dans le cadre du Carnegie Project aux États-Unis semblent confirmer des progrès à la fois en termes de motivation et en termes de résultats scolaires, lorsqu'on aide des apprenants à percevoir les origines internes d'une bonne partie de leurs résultats en apprentissage (Dickinson 1995).

Notons que l'attribution peut évoluer chez certains apprenants. La distribution des attributions en début et en fin de formation (16 mois plus tard) chez les trente apprenants de cette étude montre une augmentation des attributions internes et une régression des attributions externes, notamment externes et stables (de type « l'anglais est difficile»).

Schéma 4. Distribution d'attribution en début et fin de formation

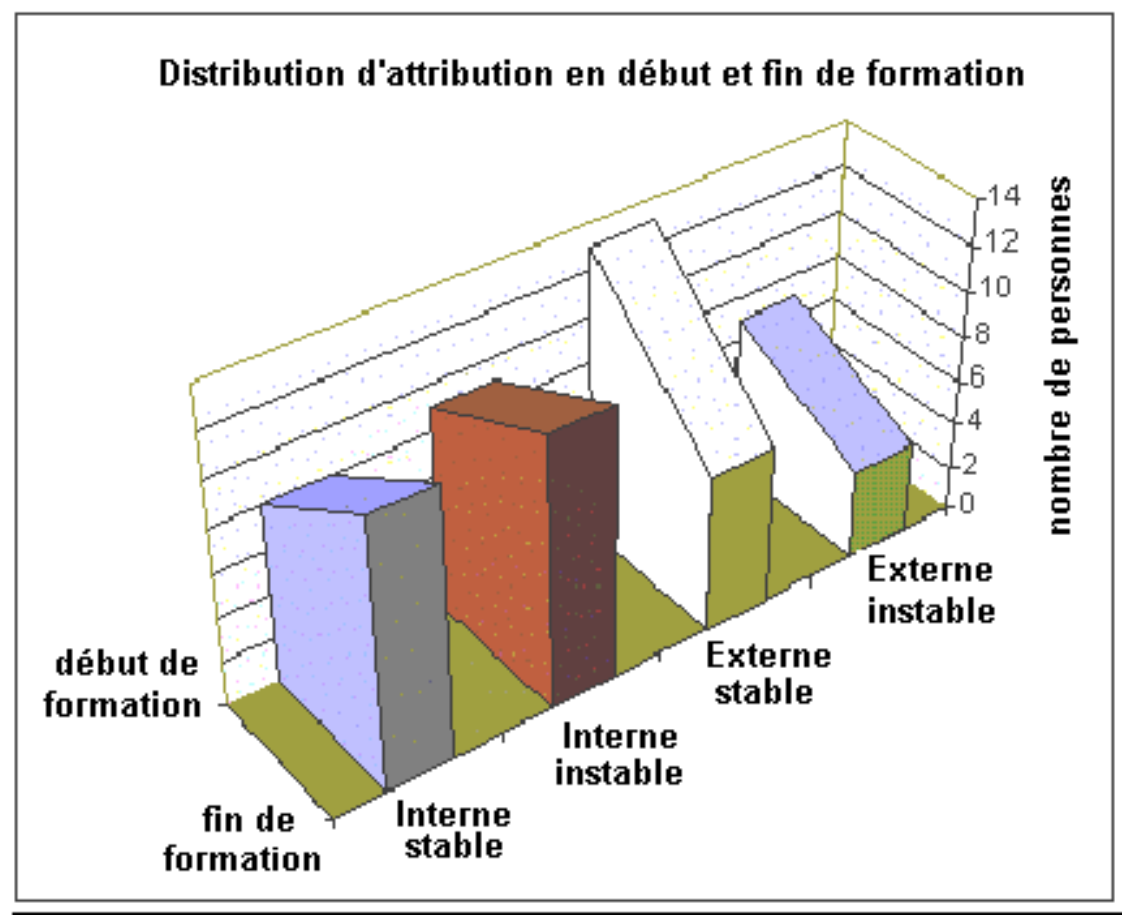

43 Cette évolution a-t-elle été influencée par la formation elle-même, par l'une des autres "différences individuelles » étudiées ou par des facteurs externes inconnus? Trop de biais, de subjectivité et de facteurs interviennent pour connaître l'origine de ces évolutions, mais la théorie de l'attribution peut néanmoins nous aider en tant que tuteurs / conseillers à amener les apprenants à des perceptions positives pour la 
poursuite de leurs apprentissages. Elle donne des indices concernant la façon d'interpréter des résultats tout au long d'une formation, afin d'instaurer une "spirale de réussite ». En somme, il vaut mieux attribuer des résultats positifs à des facteurs stables et internes et des résultats négatifs à des facteurs soit instables et internes, soit stables et externes, si l'on veut infléchir positivement l'apprentissage à venir. Il ne faut pas pour autant oublier le quatrième précepte de Locke (1981, voir « la théorie des buts ", ci-dessus) : un individu ne pourra réaliser des objectifs que dans la limite de ses capacités. Faire croire à l'impossible ne peut qu'être néfaste pour l'individu contraint par des capacités limitées.

\section{L'autodétermination}

La théorie de l'autodétermination postule qu'il existe essentiellement trois classes de motivation, la motivation intrinsèque, la motivation extrinsèque et l'amotivation. Elle fournit une grille de lecture, qui permet de situer et de faciliter la compréhension des motivations individuelles.

Schéma 5. Le modèle de l'autodétermination

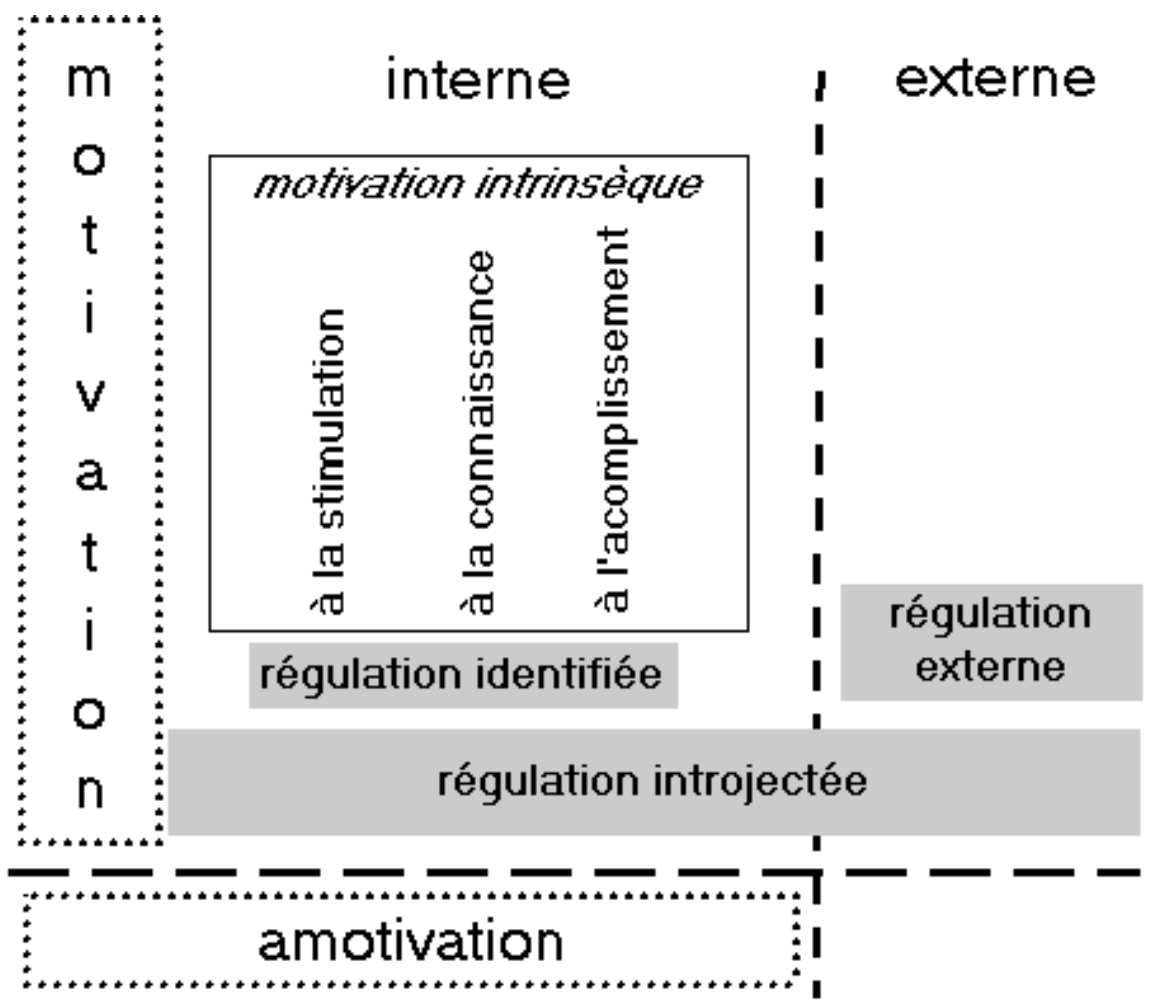

La motivation intrinsèque correspond à l'exercice d'une activité pour le plaisir et la satisfaction que l'on en retire (Deci 1975). Vallerand \& Blanchard considèrent actuellement que la motivation intrinsèque peut provenir de trois orientations distinctes :

- la motivation intrinsèque à la stimulation (le plaisir de l'activité elle-même) ;

- la motivation intrinsèque à la connaissance (le plaisir d'apprendre quelque chose ou d'explorer de nouveaux éléments) ;

- la motivation intrinsèque à l'accomplissement, ( le plaisir de produire, d'accomplir ou de créer quelque chose [1998: 20]). 
le cas de "Clara ", qui dit: «je fais de l'anglais puisque j'aime ça et quand je rencontre un anglais dans la rue, ou en vacances, je me régale à discuter avec lui ». Elle n'a pas de besoin professionnel imminent en anglais; elle déclare en faire pour sa satisfaction personnelle. Pour « Karen », qui dit aussi faire de l'anglais pour son plaisir personnel, cette satisfaction porte en revanche sur la stimulation de l'apprentissage. Elle adore venir dans le centre de langues, participer à toute activité qui lui est proposée. Elle manifeste son autodétermination dans la manière dont elle initie des séances de conseil en formation et prend en charge immédiatement leur mise en œuvre. A des niveaux de compétence langagière différents («Clara » a une maîtrise d'anglais et " Karen » est un débutant, n'ayant jamais fait d'anglais scolaire) toutes les deux montrent une motivation intrinsèque pour l'apprentissage de l'anglais. La recherche de progression personnelle est à l'origine du désir d'apprendre l'anglais. Le rôle du formateur est de préserver cet enthousiasme, en renforçant les messages de plaisir ressentis par les apprenants.

La motivation extrinsèque serait induite pour des raisons instrumentales, pour se procurer quelque chose que l'on désire ou bien pour éviter quelque chose de désagréable (Deci 1975). Deci \& Ryan (1985) ont élargi la notion de motivation extrinsèque en proposant un continuum à 5 éléments. En allant du plus probable à produire le comportement désiré, au plus improbable, nous trouvons : la motivation intrinsèque, la régulation identifiée, la régulation introjectée, la régulation externe, l'amotivation.

Cette dernière, absente des schémas précédents, rejoint la notion de résignation acquise (Abramson et al. 1978, cité dans Vallerand \& Blanchard 1998 : 21) et représente la position d'un individu "lorsqu'il ne perçoit pas de relations entre ses actions et les résultats obtenus » (Pelletier \& Vallerand 1993 : 256). Dans l'apprentissage d'une $\mathrm{L}_{2}$, un exemple serait : «j'ai fait pas mal d'anglais jusqu'ici mais dans mon boulot je ne m'en sers jamais, donc ça ne vaut plus vraiment la peine que je continue à en faire ».

Une personne motivée par régulation externe adopte un comportement en fonction de contraintes ou récompenses extérieures à elle-même. C'est la notion classique du bâton et de la carotte et elle correspond à celle qui se trouve dans la littérature sous le nom « motivation extrinsèque ». " Laurent » a une motivation de régulation externe. Il suit une formation à l'anglais puisqu'il considère avoir des impératifs professionnels qui l'exigent. Il n'en est pas particulièrement heureux; il en attend un changement de poste immanent et des promotions à terme. Ces récompenses externes justifient et nourrissent son implication. Les formateurs doivent veiller à ce que ces récompenses soient réelles et qu'ils suivront effectivement en cas de réussite. Ils doivent aussi fournir de "petites » récompenses (encouragements, «notes», reconnaissances) au fur et à mesure des réussites à chaque étape de l'apprentissage.

La régulation introjectée est un peu plus internalisée. "Patrick » manifeste ce type de motivation. Il a progressivement intériorisé les pressions ressenties par rapport à l'absence d'apprentissage de l'anglais dans le cadre scolaire et des demandes professionnelles de plus en plus insistantes. Le jour de notre entretien, il considère que l'anglais est de son ressort et il s'y implique avec une certaine détermination personnelle. Le formateurs doit être là pour l'aider, l'encourager et l'accompagner, afin de renforcer cette détermination.

51 La régulation identifiée est un niveau de motivation encore plus intériorisée et plus autodéterminée. L'origine de la motivation est externe, mais la personne l'a complètement intégrée pour son propre compte. "Jonathan » a des ambitions 
professionnelles fortes, qui exigent une maittrise de l'anglais (réunions et conduite de projets internationaux, management à l'étranger). Ces ambitions sont tellement importantes pour lui qui l'anglais est devenu non seulement un moyen pour arriver à ses fins, mais comporte un certain intérêt en soi. La motivation autodéterminée de " Jonathan » est telle que les formateurs ne doivent que peu intervenir pour le conseiller. Leur rôle est celui de ressource, que « Jonathan » vient consulter au besoin.

Dans l'ordre présenté, chacune de ces régulations représente un degré d'autodétermination supérieure à la précédente et une motivation plus forte. Ainsi, dans le cadre d'une formation autodirigée en centre de langue, on a fait l'hypothèse que plus un individu présente une motivation autodéterminée, plus il s'investira dans sa formation, notamment en termes de l'intensité et de la persistance de l'apprentissage. Pourtant, aucune corrélation statistique n'a été trouvée entre l'autodétermination et la fréquence de séances d'apprentissage (intensité) ou le nombre d'heures total de formation (persistance). Il se peut que ces interprétations $d^{\prime}$ «intensité » et de « persistance » soient trop limitées.

$\mathrm{Vu}$ d'une manière plus globale, mais aussi plus subjective, on peut considérer que l'intensité de l'apprentissage comporte aussi bien des activités en centre de langues que des actions professionnelles ou personnelles impliquant l'utilisation de l'anglais. Ainsi, dans notre échantillon, $68,7 \%$ des apprenants autodéterminés se sont impliqués dans leur apprentissage d'une manière intense, si l'on se fonde sur leurs déclarations de temps passé « en anglais » ou « sur l'anglais ». Certes, il serait intéressant d'explorer ces liens entre autodétermination, intensité et persistance d'une manière plus contrôlée; ces quelques pistes de réflexion visent à inciter des formateurs et organisateurs de centres de langues à s'appuyer sur l'autodétermination des apprenants pour faciliter leurs apprentissages.

La théorie de l'autodétermination permet d'appréhender l'intensité de la motivation, son degré d'intériorité et sa relation avec l'environnement. Elle permet de situer la motivation des apprenants suivant cinq degrés. Elle peut ainsi nous indiquer quels apprenants doivent être orientés vers d'autres activités ou apprentissages (personnes amotivées), lesquels ont besoin d'éléments externes pour avancer (et que la structure de formation pourrait fournir, au moins partiellement) et lesquels ont une motivation (intrinsèque ou de régulation identifiée) suffisante pour stimuler leur propre apprentissage.

\section{L'autodétermination et l'autonomie dans le centre de langues}

55 Ce qui distingue les différentes classes de motivation (intrinsèque, extrinsèque, amotivation) c'est le degré d'autodétermination lié à leur fonctionnement (Pelletier et Vallerand 1993). Dans un centre de langues pour adultes en entreprise, où le degré d'autonomie est assez large, et où l'on cherche à l'élargir, afin que les apprenants puissent agir seul dans le monde professionnel, cette notion d'autodétermination est très importante. Pour Deci et Ryan, c'est l'autodétermination qui mène à la motivation intrinsèque. Ainsi la création de conditions d'apprentissage informelles, qui favorisent l'autonomie, améliore la motivation intrinsèque et instaure indirectement un apprentissage plus efficace (Dickinson 1995 : 170). 
Schéma 6. Autonomie et autodétermination

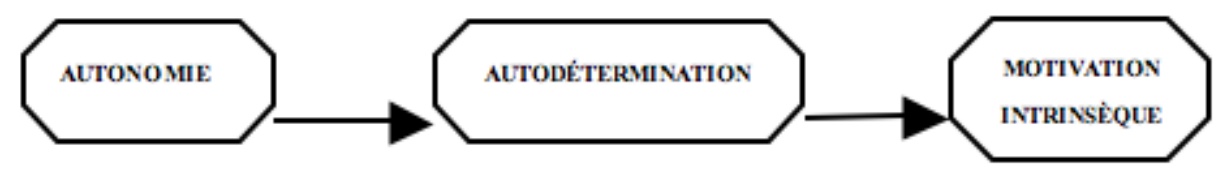

Sept apprenants de notre échantillon montrent une évolution d'autodétermination entre le début de la formation et la fin. Dans chaque cas, il s'agit d'une autodétermination plus internalisée, plus proche d'une motivation intrinsèque : de la régulation externe vers la régulation introjectée (1 personne); de la régulation introjectée vers la régulation identifiée (4 personnes); de la régulation identifiée vers la motivation intrinsèque (1 personne). Pour «Laurent ", l'intériorisation de la motivation représente deux étapes : de la régulation externe à la régulation identifiée. En effet, cet apprenant a vécu une modification radicale dans sa façon d'apprendre, mais surtout dans sa façon de se considérer vis-à-vis de l'anglais, d'appréhender des activités de communication et de prendre en charge son apprentissage. Il n'y a pas de cas, dans cet échantillon de trente, où le degré d'autodétermination aurait diminué.

\section{Schéma 7. Distribution de l'autodétermination en début et fin de formation}

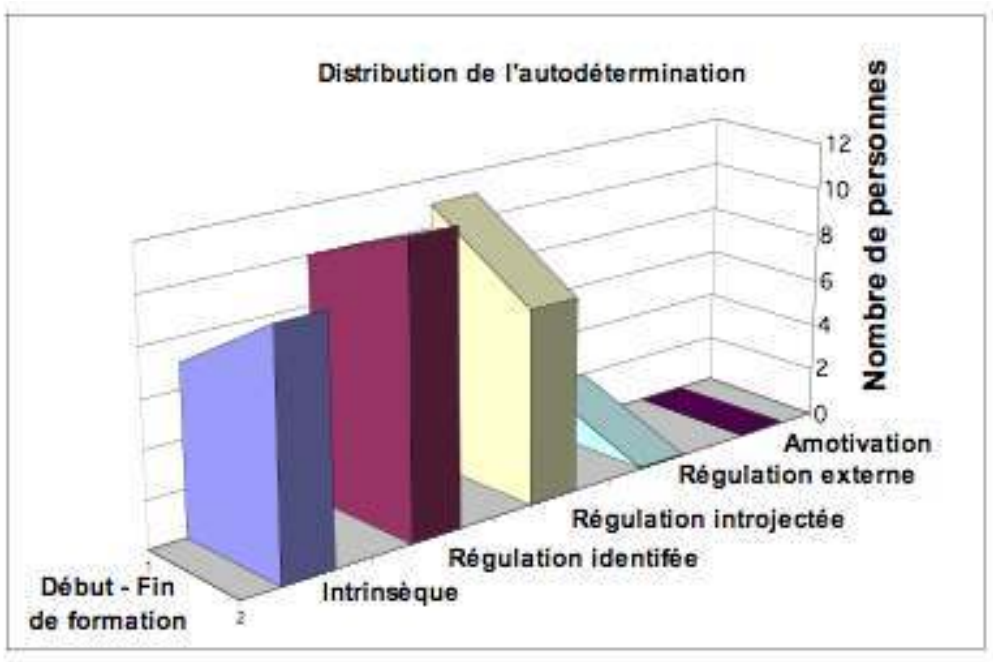

Peut-on parler d'effets d'une formation autodirigée sur la motivation intrinsèque ? Il n'y a pas de relation de cause à effet simple à établir et il n'a pas été possible d'isoler une variable qui expliquerait ces évolutions. En revanche, une hypothèse vraisemblable serait que la forme même de l'apprentissage autodirigé y a fortement contribué. Un regard sur les cas individuels tendrait à soutenir cette hypothèse. Pour «Laurent » un atelier de 4 heures semble avoir servi de déclencheur, pour qu'ensuite il prenne en charge sa propre évolution dans le centre de langues, en testant des outils variés et des organisations de travail diverses. «Émilienne » s'est servi des possibilités offertes par le centre de langues pour prendre des risques, entraîner d'autres vers les activités qui l'avaient le plus intéressée et, enfin, se convaincre qu'elle était capable de voyager seule. Ces exemples sont trop réducteurs mais pourraient inciter à réaliser des études plus ciblées sur la relation entre autonomie et autodétermination dans un centre de langues. La théorie de l'autodétermination semble indiquer une organisation en structure de formation 
autodirigée, en suggérant les effets positifs de l'autonomie en apprentissage sur la motivation intrinsèque.

\section{Alors ... motiver?} qui, par la suite, peut amener à connaître les déclencheurs spécifiques d'un individu afin de pouvoir impulser sa motivation personnelle et le conseiller pour la suite de son apprentissage.

63 Cet article met l'accent sur le déclenchement et la direction (la canalisation) de la motivation dans l'objectif d'augmenter la prise en charge personnelle de l'apprentissage par l'apprenant. Dans ce but, un travail individuel important a été réalisé au niveau de la détermination des objectifs d'apprentissage et la mise en œuvre de moyens pédagogiques adaptés dans le centre de langues. Les évolutions de motivation et d'autonomie qui ont été observées chez certains apprenants, si elles restent subjectives et relatives, peuvent néanmoins permettre de considérer que cette orientation a produit des résultats positifs.

En conclusion, la motivation ne peut être que personnelle et individuelle. C'est elle qui déclenche l'apprentissage, l'oriente, en détermine l'intensité et l'engagement dans la durée. "Motivation is best explained as a complex and dynamic process with room for several intervening variables » disent Gardner \& Tremblay (1994: 366). Il est vrai qu'elle peut se chevaucher, selon les théories, avec des notions telles que l'aptitude, les croyances, l'affectivité (cf. Toffoli 2000 : 269-299). Elle est charnière entre le cognitif et l'affectif, ce qui a même amené certains auteurs à parler de " conatif » (Carré 1997). Même si, pour 
nous praticiens, ce qui importe est l'ensemble des éléments qui participent à la réussite (ou à l'échec) d'un apprentissage, ce que Ehrman \& Oxford (1995) ont appelé « cognition plus », il semblerait qu'une attention toute particulière doive porter sur la motivation, pour son rôle fédérateur des autres éléments. Nous restons cependant persuadé que la formation des formateurs demeure un pilier central de la réussite des apprentissages. Pour les praticiens que nous sommes, la connaissance des modèles de la motivation est un gage de réussite par l'exemple.

\section{BIBLIOGRAPHIE}

Carré, P. (dir). 1998. Éducation permanente : motivation et engagement en formation. vol. 136, 3.

Carré, P., A. Moisan \& D. Poisson 1997. L'Autoformation: Psychopédagogie, ingénierie, sociologie.

Paris : Presses Universitaires de France.

Deschamps, Jean-Claude. 1993. « L'Attribution ». In Vallerand, R.J. et E. Thill (dir), Introduction à la psychologie de la motivation. Laval, Canada : Éditions Études Vivantes, 436-464.

Dickinson, Leslie. 1995. « Autonomy and motivation: A literature review ». System 23/2. 165-174.

Dörnyei, Zoltan. 1994. « Motivation and motivating in the foreign language classroom ». The Modern Language Journal 78/3, 273-284.

Dörnyei, Zoltan. 2001. Teaching and Researching Motivation. New York: Addison-Wesley Publishing Co.

Ehrman, Madeline E. \& Rebecca L. Oxford. 1995. « Cognition plus: Correlates of language learning success ». The Modern Language Journal 79/1, 67-89.

Gardner, R.C., \& P.D. McIntyre. 1992. «A student's contribution to second language learning. Part I: Cognitive variables ». Language Teaching 25, 211-220.

Gardner, R.C. \& P.F. Tremblay. 1995. « Expanding the motivation construct in language learning ». The Modern Language Journal 79/4, 505-520.

Locke, E.A., K.N. Shaw, L.M. Saari, \& G.P. Latham. 1981. « Goal setting and task performance 1969-1980 ». Psychological Bulletin 90, 125-52.

Mager, R. F. 1984. Goal Analysis. Belmont, CA : Lake Publishing Co.

Mager, R. F. 1984. Preparing Instructional Objectives. Belmont, CA : Lake Publishing Co.

Nelson, Marie Wilson. 1991. At the Point of Need: Teaching basic and ESL writers. Portsmouth, NH : Boynton/Cook Publishers.

Nunan, David. 1988. The Learner-centered Curriculum. Cambridge : Cambridge University Press. Nunan, David. 1995. « Closing the gap between learning and instruction ». TESOL Quarterly 29/1, 133-158.

O’Bryen, Patricia. 1996. « Using questionnaires to assess motivation in second language classrooms ». University of Hawaii Working Papers in ESL 14/2, 73-125. 
Oller, J.W. Jr. 1986. « Communication theory and testing: What and how », in Stansfield, C.W. (ed.), Toward Communicative Competence Testing: Proceedings of the Second TOEFL Invitational Conference. Princeton: ETS.

Oxford, Rebecca \& Jill Shearin. 1994. « Language learning motivation: Expanding the theoretical framework ». The Modern Language Journal 78/1, 12-28.

Skehan, Peter. 1989. Individual Differences in Second-Language Learning. Londres : Edward Arnold.

Toffoli, Denyze. 2000. « Au cœur de la formation : l'apprenant (une recherche-action sur l'apprentissage de l'anglais en milieu professionnel) ». Thèse de doctorat, Université de la Rochelle.

Vallerand, R. J. \& C. Blanchard, 1998. «Éducation permanente et motivation : contribution du modèle hiérarchique de la motivation intrinsèque et extrinsèque ». Éducation Permanente 136/3, 15-36.

Vallerand, R. J. \& E. Thill (dir.). 1993. Introduction à la psychologie de la motivation. Laval, Canada : Éditions Études Vivantes.

Williams, Marion. 1994. « Motivation in foreign and second language learning: An interactive perspective ». Educational and Child Psychology 11/2, 77-84.

\section{NOTES}

1. Les prénoms sont fictifs, mais les exemples présentés sont tous réels.

\section{RÉSUMÉS}

Comment mettre en œuvre différentes théories cognitives de la motivation dans un centre de langues? En partant de quelques définitions de la motivation dans le cadre de la formation, cet article retrace une recherche-action au sein d'un centre de langues dans une entreprise française. Les théories des buts, de la V.I.E. et de l'auto-efficacité, permettent de décrire un processus d'entretien qui en a découlé pour la détermination des parcours individuels de formation dans le centre. La théorie de l'attribution a servi à ajuster l'interprétation des apprentissages antérieurs de manière à essayer de positivement influencer l'avenir. Enfin, le fonctionnement même d'un centre de langues autodirigé, qui préconise les interventions formatives en fonction du degré d'autodétermination de l'individu permet d'accroître les facteurs de motivation. Bien évidemment cela implique l'intervention de formateurs spécifiquement formés aux techniques décrites. La motivation, comme d'autres paramètres cognitifs et affectifs de l'individu, dépend de l'individu, mais peut être influencé par des éléments externes.

This article presents practical applications of cognitive theories of motivation in a language centre. After citing some pertinent definitions of motivation for education, this article follows an action-research in a self-access language centre in a French company. Working from theories of goal setting, V.I.E. and self-efficacy, an interview process was set-up in order to define 
individualised training programmes in the centre. After explaining this motivational process, attribution theory indicates how learners can interpret the results of prior language learning experiences in order to influence future learning positively. Finally, the functioning of the selfdirected learning centre itself, by basing training prescriptions on the degree of students' selfdetermination, also contributes to enhancing motivation. This obviously requires specialised trainers well versed in the techniques described. Like other cognitive and affective factors, motivation depends on the individual, but can be influenced by external elements.

\section{INDEX}

Mots-clés : attribution, auto-efficacité, autodétermination, but, théorie cognitive de la motivation, V.I.E.

Keywords : cognitive theory of motivation, goal, self-determination, self-efficacy

\section{AUTEUR}

\section{DENYZE TOFFOLI}

Denyze Toffoli dirige Development \& Training, une agence de recherche, de conseil et de formation en langues, depuis sa création en 1992. Elle y développe des centres de formation autodirigés, des séminaires ludiques pour " apprendre à apprendre ", des doubles apprentissages professionnels et des formations de formateurs. Titulaire d'un doctorat (2000) en didactique de l'anglais sous la direction de J-P Narcy-Combes, ses domaines de recherche sont l'individualisation de l'apprentissage des adultes en entreprise, le développement de l'entreprise via la formation, la conjonction cognitif/affectif dans l'apprentissage. dtoffoli@unistra.fr 